Assessing the Difficulty of Mathematical Translations: Synthesizing the Literature and Novel Findings

\author{
Michael J. Bossé, Kwaku Adu-Gyamfi, \& Meredith R. Cheetham \\ East Carolina University
}

\begin{abstract}
Students perennially demonstrate difficulty in correctly performing mathematical translations between and among mathematical representations. This investigation considers the respective difficulty of various mathematical translations based on student activity (defining mathematical errors during the translation process, teacher beliefs and instructional practices, student interpretive and translation activities, and the use of transitional representations) and the nature of individual representations (fact gaps, confounding facts, and attribute density). These dimensions are synthesized into a more complete model through which to analyze student translation work and delineate which mathematical translations are more difficult than others.
\end{abstract}

Keywords: Representations, translations, difficulties, student errors

The generic terms translation and translation process refer to the psychological, intellectual, or cognitive processes subsumed in transforming information encoded in one mathematical representation (source) to another (target) (Janvier, 1987). Students perennially demonstrate difficulty in translating among verbal, tabular, graphical, and algebraic mathematical representations. Moreover, the literature reports of many types of errors students make when translating among these representations including total equation errors, product errors, reversal errors, slope/height errors, interval/point errors, discrete/continuous errors, iconic/syntactic errors, interpretation errors, preservation errors, and implementation errors (Adu-Gyamfi, Stiff, \& Bossé, 2012; Bell, Brekke \& Swan, 1987; Preece, 1993).

Numerous studies have investigated components surrounding the translation process (e.g., Knuth, 2000), student successes and failures (e.g., Duval, 2006), techniques employed by students (e.g., Janvier, 1987; Pyke, 2003), and variations in the translation process by differing ability levels of students (e.g., Bossé, Adu-Gyamfi, \& Cheetham, 2011a; Brenner, Herman, Ho, \& Zimmer, 1999; Gagatsis \& Shiakalli, 2004). Altogether, unfortunately, the vast majority of these studies remains disconnected from others and mostly expresses the fact that students have difficulty with particular translations without clearly articulating why this might be (Superfine, Canty, \& Marshall, 2009).

The purpose of this paper is to perform an extensive synthesis of existing literature and integrate such with novel findings in order to generate a model to concretize notions regarding the respective difficulty of each translation between mathematical representations. To accomplish such, we consider numerous dimensions reported in the literature, further these findings with novel research, and seek to assess student difficulties based on the literature and previously undeveloped connecting constructs. 


\section{Structure of the Paper}

In order to develop the internal argument and present the findings of this investigation, this paper is structured in the following manner. First, it is demonstrated that the difficulty of respective mathematical translations can be assessed on two dimensions: student-centered factors and content- or representation-centered factors. Second, numerous dimensions regarding the translation process (e.g., student translation errors, teacher beliefs and instructional practices, translation actions, and the use of transitional representations) are considered as they affect the respective difficulty of each translation. Third, dimensions regarding the interpretive activity surrounding individual mathematical representations (e.g., fact gaps, confounding facts, and attribute density) are investigated in respect to their affect upon the translation process. As an amalgamation, these dimensions paint a picture of the multifaceted nature of translations and the difficulties students encounter with such. Fourth, the authors briefly describe some of their previous research, which led to questioning whether some mathematical translations are more difficult than others. Fifth, all the preceding discussions are synthesized under novel constructs in a manner, which organizes and orders each translation in respect to its level of difficulty.

\section{Defining the Difficulty of Mathematical Translations}

Differentiating difficulty levels among various translations can consider numerous dimensions. These dimensions can be generalized to student-centered factors and contentcentered factors. Regarding student-centered factors, one can investigate the actions students take when performing translations. Since these actions differ among translations (Janvier, 1987), it is conceivable that some of these actions are more difficult than others. Second, students often utilize dual translations as they begin with a source representation, translate to a transitional representation, and then translate the transitional representation into a target representation (Bossé, Adu-Gyamfi, \& Cheetham, 2011b). The employment of dual translations adds another dimension of difficulty to some translations. Third, since concepts and tasks which are frequently experienced through instruction are mastered more readily than those which are less frequently experienced, it is understandable that classroom experiences may diminish the perceived level of difficulty of translations more frequently experienced and accentuate the perception of difficulty among translations less frequently experienced (Bossé et al., 2011b).

Within the content- or representation-centered factors, additional issues are at play, which may affect the assessment of the difficulty level of respective translations. First, some representations require different interpretive techniques than others. This may lead to differing levels of difficulty. Second, some translations are inherently more complex, requiring greater conceptual understanding than others, and some require a greater number of steps in the translation process. These two factors are further examined herein.

Altogether, the assessment of which translations are more difficult than others is necessarily an amalgam of these student- and content-centered translation factors and is, therefore, non-trivial. While all singleton factors introduce some valuable understanding of the nature of the translation process and the difficulties associated with each, independently 
these do not paint a sufficient picture to fully assess the difficulty level of each translation. Thus, in this study, these factors are initially considered independently and then the gestalt of these dimensions is considered to establish a more encompassing evaluation of the level of difficulty of each translation.

\section{Dimensions Regarding Mathematical Translations}

In the discussions, which immediately follow, we consider the nature of, and difficulty associated with, mathematical translations based upon documented literature on student centered factors (i.e., errors, teacher beliefs and instructional practices, interpretive and translation activities, and translations that require use of transitional representations). For the most part, this literature creates an argument that some mathematical translations are more difficult than others. Fuller analysis, synthesis, and discussion of the differing levels of difficulty of various mathematical translations follow in later sections.

\section{Student Errors in Translating among Numeric, Algebraic, and Graphical Representations}

Students perennially demonstrate difficulty in translating among numeric, graphical, and algebraic representations of associated mathematical relations (Dreyfus \& Eisenberg, 1987; Dunham \& Osborne, 1991; Gagatsis \& Shiakalli, 2004; Galbraith \& Haines, 2000; Kieran, 1993; Knuth, 2000; Macgregor \& Stacey, 1993; Porzio, 1999). Moreover, the literature reveals different types of errors students make when translating among these representations, including: manipulation errors, where the student computes an arithmetic/algebraic problem incorrectly or utilizes variable names incorrectly, and conceptual errors, where a student either introduces an incorrect constraint (errors of commission) or overlooks a critical constraint (errors of omission). Some of these errors take the form of slope/height confusion errors, interval/point confusion errors, discrete/continuous confusion errors, and iconic/syntactic confusion errors (Bell, Brekke, \& Swan, 1987; Kerslake 1987; Preece, 1993).

For example, in a study investigating student actions during translations between numeric, symbolic, and graphical mathematical representations, Adu-Gyamfi et al. (2012) found three distinct types of common student errors: interpretation errors, implementation errors, and preservation errors. Figure 1 denotes both the type of error observed and between which pair of representations the errors occurred. In Figure 1, the arrows depict the direction of the translation (e.g., under Implementation Error, Symbolic $\rightarrow$ Table denotes that a symbolic representation is to be translated into a tabular representation) and the numeral on each arrow denotes the frequency of occurrence of the respective type of error as reported in the study. Therefore, for example, as students attempted to translate from a graph to a table, 11 interpretation errors were observed from the cases of student work analyzed.

Interpretation error "arises when the student incorrectly ascribes, characterizes, or exemplifies attributes or properties of either the source or target representation." For instance, a student may incorrectly read the point $(5,-3)$ on a graph as the ordered pair $(-3,5)$. Implementation error denotes incorrectly executed steps, computations, and algorithms employed in the process of translating from one representation to another. For example, a student may substitute a value for $y$ in an equation and solve for $x$, when it would have been 
correct to substitute the value for $x$ and solve for $y$. Preservation error occurs when "the student correctly maintains semantic congruence between the source and target representations for self-identified attributes or properties, but fails to confirm that other relevant attributes or properties are also correctly translated." For instance, a student may recognize that a linear equation has a slope of $1 / 2$ but may create an incorrect corresponding graph with a slope of $2 / 1$, without sufficiently comparing the two representations.

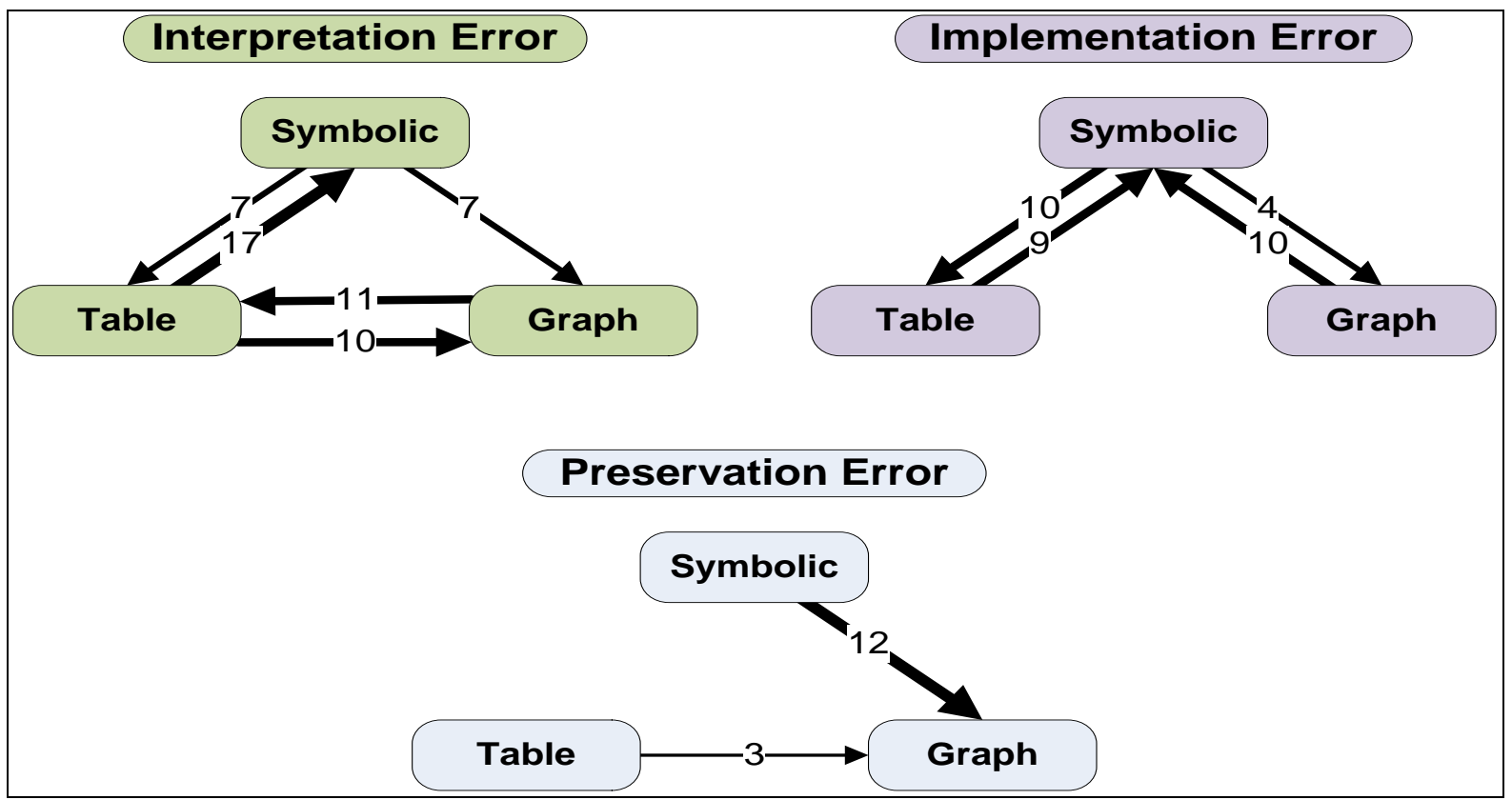

Figure 1. Overview of findings from Adu-Gyamfi, Stiff, and Bossé (2012).

\section{Student Errors in Translating from and to Verbal Representations}

In translation situations which begin in verbal form and are translated into symbolic representations, such as the students-professors problem (Clement, Lochhead \& Monk, 1981) and Mindy's restaurant problem (Wollman, 1983), two consistent types of error processes have been identified from interview protocols: word order matching process and static comparison process. In word order matching, the student simply assumes that the order of key words in the problem corresponds to the order of the symbols appearing in the equation. Clement (1982) referred to this approach as a syntactic strategy, because it does not depend on the meaning of the expression. The static comparison process, in contrast, does consider the meaning of the expression and entails realizing that there are more students than professors but not knowing how to express this relationship.

When tabular, symbolic, or graphical representations are translated into verbal form, additional dimensions arise which differentiate these from other translations (Gagatsis \& Shiakalli, 2004; Janvier, 1987; Kerslake, 1981; Roth \& Bowen, 2001; Wainer, 1992). First, however, it is necessary to define what is meant by translating to a verbal representation. In this investigation, verbal mathematical representations are defined as either verbal situations or verbal descriptions. Verbal situations are best defined as real world scenarios depicted verbally (e.g., "Jane and John are each carrying packages. Jane's package weighs $5 \mathrm{~kg}$ and John's package weighs $10 \mathrm{~kg}$...”). Verbal descriptions are characterizations in verbal form of mathematical representations in symbolic, tabular, or graphical form (e.g., "the function is a 
polynomial with zeros at ..."). Although "verbal representation" will herein always denote the situational scenario when it is the source and the descriptional scenario when it is the target, when necessary, these scenarios will be differentiated using the obvious denotations "verbal description" and "verbal $_{\text {situation". }}$

Beyond the fact that students more often struggle to perform translations from nonverbal to verbal representations and that there is variability in the definition for a target verbal representations, evidence demonstrates that these translations carry other factors which make them inherently more difficult than other mathematical translations. Bossé et al. (2011b) state that when students attempt to translate from graphs, tables, and symbolic representations to verbal form, they are rarely able to do so beyond the context of simple linear functions or simple exponential functions. When the relationship depicted in the non-verbal source representation is that of numerous other functions (polynomials, logarithmic, sinusoidal, and etc.), students are generally unable to capture the relationship in a verbal situation or word problem (Carlson, 1998; Kaput, 1989). Unfortunately, because these functions are more complex, it is difficult to determine if students' failures are associated with the increasing complexity of the mathematics or their inability to perform these translations.

\section{Instructional Practices and Teacher Beliefs}

Summarily, when the total number of errors is considered irrespective of the particular type of representations being translated, the work of almost all previously cited studies reveals, either tacitly or explicitly, that students experience difficulties with some translations more than others. The question naturally arises as to why students struggle with certain types of mathematical translations. In the discussions, which immediately follow, we consider the nature of, and difficulty associated with, performing mathematical translations based upon instructional techniques and teacher beliefs, as deciphered from the extant literature.

In order for students to succeed in any mathematical translation, they must be able to work with configurations of text, syntax, and characters of given source and target representations. They must also be able to automatically switch from syntactically processing these configurations to semantically processing these configurations and vice versa (Kaput, 1989). Since these skills or processes do not automatically develop, there is a need for a classroom culture where opportunities are provided for students to not only perform these translations but to also have their translation difficulties diagnosed and addressed. Subsequently, curricular and instructional emphasis has been identified as one of the most significant mitigating factors to students' difficulties as well as errors in mathematical translations (Cunningham, 2005; Knuth, 2000).

Surveys and observations of instructional practices reveal that teachers heavily emphasize some translations (symbolic $\rightarrow$ graph, symbolic $\rightarrow$ table, table $\rightarrow$ graph, and graph $\rightarrow$ table), give significantly lesser attention to other translations (verbal $\rightarrow$ symbolic, verbal $\rightarrow$ table, table $\rightarrow$ symbolic, and graph $\rightarrow$ symbolic), and almost completely avoid some translations (symbolic $\rightarrow$ verbal, table $\rightarrow$ verbal, and graph $\rightarrow$ verbal) with the exception that students were to verbally define what they observed in a table, graph, or symbolic expression (e.g., Bossé et al., 2011b; Cunningham, 2005; Porzio, 1999). Clement et al. (1981) note that students are rarely asked to generate a formula from a given verbal representation but are usually asked to select one from a well-defined list and to manipulate it. Altogether, it becomes immediately 
apparent that a connection may exist between student errors and teacher instructional practices in regards to mathematical translations. That is, teachers provide the least number of opportunities for students in translations where students have the most difficulty.

Additionally, researchers have also found significant correlations among student ability with various translation and teacher expectations regarding which translations students should be able to correctly perform. Numerous studies (e.g., Prozio, 1999; Schoenfeld, Smith \& Arcavi, 1993) find that secondary Algebra and Pre-calculus teachers believe that students are generally successful in some translations (verbal $\rightarrow$ table, symbolic $\rightarrow$ graph, symbolic $\rightarrow$ table, table $\rightarrow$ graph, and graph $\rightarrow$ table), less successful in others (verbal $\rightarrow$ symbolic, table $\rightarrow$ symbolic, and graph $\rightarrow$ symbolic), and rarely successful in others (symbolic $\rightarrow$ verbal, table $\rightarrow$ verbal, and graph $\rightarrow$ verbal). Teachers report that, to some extent, students are generally able to verbally describe attributes and characteristics found in graphs, tables, and functions $\left(\right.$ graph $\rightarrow$ verbal $_{\text {description, }}$ table $\rightarrow$ verbal $_{\text {description, }}$, and symbolic $\rightarrow$ verbal $1_{\text {description }}$ ) (Bossé et al., 2011b). Thus, to the degree that verbally describing other representations is considered translating to a verbal form, students are often successful. Additionally, students are generally able to perform the verbal $\rightarrow$ graph translation, albeit by employing a transitional representation (usually verbal $\rightarrow$ table $\rightarrow$ graph). However, this generally falls well short of translating any nonverbal representation into a verbal representation in the form of a problem scenario or situation. These translations $\left(\right.$ graph $\rightarrow$ verbal $_{\text {situation, table } \rightarrow \text { verbal }_{\text {situation, }} \text {, and }}$ symbolic $\rightarrow$ verbal $\left._{\text {situation }}\right)$ are generally considered most difficult and are least often performed successfully by students (Mevarech \& Kramarsky, 1997; Monk, 1992; Roth \& Bowen, 2001).

Altogether, it could be argued that student difficulty with particular translations may be correlated to the fact that teachers believe (or do not believe) that students can perform such and, may therefore, emphasize (or de-emphasize) experiences with these translations during classroom instruction. However, the literature indicates that placing the onus singularly on teacher beliefs and instructional practices may not sufficiently account for difficulties students experience with certain translations (e.g., Resnick \& Omanson, 1987; Yerushalmy, 1991). For example: Webb (1990) reports that students who had the most classroom practice translating between verbal and numerical representations and between algebraic equations and verbal descriptions showed the worst performance on problems of these types; Clement et al. (1982) suggest a focus on the translation skill itself in order to better understand the nature and source of student difficulty; and Leinhardt, Zaslavsky and Stein (1990) identify two types of activities involved in a translation: interpretation and construction. In the following discussion, these two activities will be further explicated in respect to student translation difficulties.

\section{Constructive Activity (Translation Action)}

Construction refers to the action of generating new parts that are not given, such as building a graph from a function rule or a table. Each construction activity, when considered in the context of a translation, is uniquely associated with an action, technique, or heuristic (e.g., curve fitting and sketching) that specifies how constructs or descriptions expressed in the source representation can be directly articulated through structures available in the target representation (Galbraith \& Haines, 2000; Lesh, Landau, \& Hamilton, 1983). A list of construction activities, denoted by Janvier (1987) as translation actions, between each type of 
representation is provided in Figure 2. Since each of these construction activities denotes a different skill (and some are vastly dissimilar), some of these translation skills differ in complexity, and possibly difficulty (Duval, 2006). Therefore, the type of action associated with a translation may play a role in the difficulty of the translation (whether causal or correlational). The question naturally arises as to the role of these actions in respect to student errors during translations.

\begin{tabular}{|l|c|c|c|c|}
\hline From & $\begin{array}{c}\text { Situations, Verbal } \\
\text { Description }\end{array}$ & Table & Graph & $\begin{array}{c}\text { Formulae } \\
{[\text { Symbolic }]}\end{array}$ \\
\hline $\begin{array}{l}\text { Situations, Verbal } \\
\text { Description }\end{array}$ & & Measuring & Sketching & Modeling \\
\hline Table & Reading & & Plotting & Fitting \\
\hline Graph & Interpretation & Reading off & & Curve fitting \\
\hline Formulae [Symbolic] & Parameter Recognition & Computing & Sketching & \\
\hline
\end{tabular}

Figure 2. Translation actions (adapted from Janvier, 1987).

Altogether, initial correlation seems to exist between the translation action and the level of student success in the respective translations. For instance, students seem generally more able to utilize some translation techniques

verbal $\underset{\text { measuring }}{\rightarrow}$ table, symbolic $\underset{\text { sketching }}{\rightarrow}$ graph, table $\underset{\text { plotting }}{\rightarrow}$ graph, and graph $\underset{\text { reading off }}{\rightarrow}$ table

and less successful in other translation techniques

symbolic $\underset{\substack{\text { parameter } \\ \text { recognition }}}{\rightarrow}$ verbal, table $\underset{\text { reading }}{\rightarrow}$ verbal, graph $\underset{\text { interpretation }}{\rightarrow}$ verbal, and verbal $\underset{\text { sketching }}{\rightarrow}$ graph .

Arguably, computing, reading off, and plotting, are less complex techniques than are sketching, curve fitting, and fitting (Janvier, 1987). Additionally, sketching as associated with the symbolic $\rightarrow$ graph translation and sketching as associated with verbal $\rightarrow$ graph translation have both similarities and dissimilarities. Unfortunately, apart from correlation with the frequency of success or failure of the various translations (Adu-Gyamfi et al., 2012), little direct analysis of the varying difficulty of each of these translation techniques is available.

\section{Interpretative Activity (Proximity)}

Interpretation refers to the action by which a student makes sense of, or gains meaning from, a representation (Leinhardt et al., 1990; Wainer, 1992). Researchers argue that, since a one-to-one relationship cannot always be established between information associated with source and target representations in a translation, interpretation of facts within each representation and across representations has to be made in order to resolve ambiguities. For example, in order to construct a graph for a given set of ordered pairs, a translator must necessarily know that to every ordered pair of numbers in the set one can associate a point on a coordinate plane. This interpretative act is described by some researchers as a local 
interpretation (e.g., Leinhardt et al., 1990). However, in order to construct a symbolic rule for a given set of ordered pairs, a translator must not only know how the coordinates of each ordered pair are changing in relationship to each other but must also recognize the overall variation occurring among the ordered pairs of the set. This type of interpretative behavior is described as a global interpretation (Duval, 2006).

Many researchers postulate a connection between the nature of student errors in translations and the type of interpretive activity required in such translations; students have more difficulty and perform more errors in respect to translations requiring global as opposed to local interpretive actions (e.g., Dreyfus \& Eisenberg, 1987; Dunham \& Osborne, 1991; Gagatsis \& Shiakalli, 2004). Figure 3 depicts the local or global interpretive activities associated with each translation (Adu-Gyamfi et al., 2012; Knuth, 2000). In this figure, the left hand column denotes the form of the source representation and the top row denotes the form of the target representation in a respective translation. For instance, to perform the translation from graph to a table requires a local interpretive activity and to translate from a graph to a formula requires a global interpretive activity.

\begin{tabular}{|l|c|c|c|c|}
\hline From & $\begin{array}{c}\text { Situations, Verbal } \\
\text { Description }\end{array}$ & Table & Graph & $\begin{array}{c}\text { Formulae } \\
{[\text { Symbolic }]}\end{array}$ \\
\hline $\begin{array}{l}\text { Situations, Verbal } \\
\text { Description }\end{array}$ & Global & Global & Global & Global \\
\hline Table & Global & Local & Local & Global \\
\hline Graph & Global & Local & Local & \\
\hline $\begin{array}{l}\text { Formulae } \\
\text { [Symbolic] }\end{array}$ & & & & \\
\hline
\end{tabular}

Figure 3. Interpretive activity associated with translations.

Interestingly, as depicted in Figure 3, when a translation is reversed, the nature of the interpretive action can be significantly different. For example, while the table $\rightarrow$ plotting graph translation requires a local interpretive action involving a one-to-one mapping between constituent elements of the table to the graph, the graph $\rightarrow$ reading table translation requires a global interpretation action guided by understanding of qualitative variables (Duval, 2006).

\section{Transitional Representations}

While each translation action from one representation to another is singularly denoted, it has been recognized that various translations necessitate more than one constructive activity or process. While Janvier (1987) signifies translations requiring more than one process as "indirect processes" (as opposed to "direct processes"), Bossé et al. (2011a, 2011 b) use the term "transitional representation" to indicate that the translation process from source to target representation includes two translation actions and passes through an intermediary representation. For instance, in performing three translations (verbal $\rightarrow$ graph, 
symbolic $\rightarrow$ graph, and verbal $\rightarrow$ symbolic), students generally utilize transitional representation and perform the compound translations (including the dimension of proximity):

$$
\text { verbal } \underset{\text { measuring }}{\stackrel{\text { global }}{\rightarrow}} \text { table } \underset{\text { plotting }}{\stackrel{\text { local }}{\rightarrow}} \text { graph ; verbal } \underset{\text { measuring }}{\stackrel{\text { global }}{\rightarrow}} \text { table } \underset{\text { fitting }}{\stackrel{\text { local }}{\rightarrow}} \text { symbolic }
$$

and

$$
\text { symbolic } \underset{\text { computing }}{\stackrel{\text { local }}{\rightarrow}} \text { table } \underset{\text { plotting }}{\stackrel{\text { local }}{\rightarrow}} \text { graph . }
$$

Notably, then, these compound translations are mechanisms for converting global interpretive activities into local interpretive activities.

This leads to a different means for evaluating the difficulty of particular translations. Previously, dimensions such as the translation action, the proximity of interpretive activity, and the nature of student errors were considered to shed light on the potential difficulty of each translation. However, it is only conceivable that translations that require a transitional representation (and therefore more than one translation action) may carry some additional degree of complexity and potentiality for error. Indeed, the very translations that require transitional representations (verbal $\rightarrow$ graph, symbolic $\rightarrow$ graph, and verbal $\rightarrow$ symbolic) are the selfsame ones which most researchers and teachers report as most problematic to students and which teachers are most reluctant to cover in class instruction.

\section{First Summary}

From a number of dimensions (e.g., student errors, teacher beliefs and instructional practices, translation actions, proximity of these actions, and transitional representations), the literature reveals that students find some mathematical translations are more difficult than others. Synthesizing the previous research, five levels of difficulty are recognizable among the different translations: easy (table $\rightarrow$ graph, graph $\rightarrow$ table, symbolic $\rightarrow$ table, and verbal $\rightarrow$ table); easy and using transitional representations (symbolic $\rightarrow$ graph); more difficult (table $\rightarrow$ symbolic and graph $\rightarrow$ symbolic); more difficult and using transitional representations (verbal $\rightarrow$ graph and verbal $\rightarrow$ symbolic); and most difficult (graph $\rightarrow$ verbal, symbolic $\rightarrow$ verbal, and table $\rightarrow$ verbal). This ordering of difficulty will be further investigated through additional dimensions of representations in the following discussions. Previously, the activity of instruction and the nature of the translation skill (the translator) were central; in the following discussion, the nature of the representations involved in the translation is primary.

\section{Representations}

In this paper, the term representation (mathematical representation) is used in reference to external objects, such as tables, graphs, verbal expressions, or algebraic (or symbolic) depictions, whose relationship with the mathematical object or concept they signify is established through shared mathematical conventions. When individual representations are considered in respect to student difficulties with translations, a number of dimensions begin 
to emerge, including: fact gaps, attribute density, and confounding concepts (Adu-Gyamfi et al., 2012; Adu-Gyamfi et al., 2011a, Bossé et al., 2011a). Altogether, these dimensions, along with the numerous dimensions previously listed, paint a clearer picture regarding the varying levels of difficulty among mathematical translations.

\section{Fact Gaps}

Each mathematical representation contains missing information particularized to certain contexts. For instance, given a table of values representing a function, the $x$ - and $y$-intercepts may or may not be present in the data. If the context for examining the table is to determine if the relationship depicted represents a function, the absence of these entries in the table have little effect on this determination and do not represent fact gaps associated with this investigation. However, if the table is to be investigated to find $x$ - and $y$-intercepts and these values are not in the table, these represent fact gaps for this representation under this context.

Notably, in some instances, representations can be transformed to remove fact gaps. For instance, the quadratic $x^{2}+x-6$ can be written as $(x+3)(x-2)$ or $(x-1 / 2)^{2}+23 / 4$ (which will herein be denoted as different forms of the same representation). Thus, depending on the given form, the quadratic reveals its coefficients and its graph's concavity, the factors and zeros are evident, or the vertex is recognized. Associated with most representations are techniques for transforming from one form to another in order to alter the fact gaps: equations can be rewritten; table rows can be reordered; verbal representations can be restated; and one can zoom in or out to particular regions on a graph. Altogether, a student's understandings of both the representation and techniques for transforming such can affect his ability to fill fact gaps and more efficiently translate from a source to a target representation.

The number of fact gaps associated with any representation can only be determined in conjunction with considering three factors: the purpose for inspecting the representation (or the translation being attempted); whether or not the student is able or allowed to transform the source representation before attempting the translation; and whether the representation acts as a source or a target in the translation. This latter factor produces interesting results. For instance, when a table is a source representation and is to be translated into an equation, the table may contain numerous fact gaps (and indeed provide insufficient information to allow the translation to occur), the type of function may be indistinguishable, and the zeros of the function may not be provided. However, when the translation is from an equation to a table, the table will contain very few fact gaps, in that it can contain as many elements as the student wishes to compute from the equation.

The number of fact gaps associated with either the source or target representation involved in a translation may speak to the difficulty of the translation (Adu-Gyamfi et al., 2012; Bossé et al., 2011a). Students may perceive translations rife with fact gaps as more difficult than translations with fewer fact gaps.

\section{Confounding Facts}

Through revealed facts, attributes, and characteristics, every mathematical representation depicts concepts; however, most representations can also potentially contain confounding facts in respect to the desired translation. For instance, while the graph of a polynomial function may include numerous relative maxima and minima (singletons between each real 
zero), all of this information may be both unnecessary and confounding as students attempt to translate the graph into some form of an equation. Additionally, if data in a table is not presented in sequential order in respect to $x$ or if the table depicts numerous ordered pairs in which different $x$ values are associated with the same $y$ value, students may become confused by the data and struggle to plot such on a graph.

As with fact gaps, the number of confounding facts associated with any representation can only be determined in respect to: the purpose for inspecting the representation (or the translation being attempted), whether or not the student is able or allowed to transform the source representation before attempting the translation, and whether the representation acts as a source or a target in the translation. For instance, when a graph (source) is being translated into an equation (target), the graph may potentially possess numerous characteristics, which may perturb the translation process and produce more confusion than assistance. However, when a table of values is being plotted onto a graph, apart from potentially understanding the scaling of the axes, little information in the graph should hinder the student's performing of the translation and the students should be confused by very little.

The number of confounding facts associated with either the source or target representation involved in a translation may speak to the difficulty of the translation (AduGyamfi et al., 2012; Bossé et al., 2011a). Students may have more difficulty interpreting each representation and performing the translation action between representations when numerous confounding facts are present.

\section{Attribute Density}

Adu-Gyamfi et al. (2012) define attribute density as a characteristic of mathematical representations, which considers simultaneously how much information a representation provides, and how much effort is required to find additional information in a representation. For instance, both tables and verbal representations provide limited information and unearthing more information from these representations can involve significant work. Equations and graphs provide a rich amount of information and unearthing more information is often simply a case of transforming the equation into another symbolic form or zooming onto different parts of the graph. Thus, from least dense to most dense, mathematical representation are organized in the order of:

$$
\underbrace{\text { table and verbal }}_{\text {low density }} \text { and } \underbrace{\text { graph and equation }}_{\text {high density }} \text {. }
$$

As with fact gaps and confounding facts, albeit more stable and less affected by whether the representation serves as a source or target in a translation, attribute density still contains some of these concerns. For instance, although it may be difficult to discern any additional information from a table apart from what is immediately available in such, as a target this is rarely necessary. Data from a verbal, symbolic, or graphical representation can be entered into the table without much need for attempting to discern more information from the table. Thus, as a table alters from source to target, its density may change slightly; but because the information it contains is still limited, the density of a table remains low. Other representations seem, however, even less affected by the source/target directionality. 
Attribute density seems to have some impact on the difficulty of some translations over others. As will be further developed in the following discussion, the interconnection of all preceding dimensions will provide the most complete picture of which translations are more difficult than others.

\section{Second Summary}

Previously, this paper considered the respective difficulty of various mathematical translations based on student activity. Some of these considerations included, defining mathematical errors during the translation process, teacher beliefs and instructional practices, student interpretive and translation activities, and the use of transitional representations. Through these dimensions, some translations were recognized as more difficult and error prone than others. In this section, the nature of individual representations is recognized to play additional roles in determining which translations are more difficult than others; translations that involve source representations with high fact gaps and confounding facts and low attribute density lead to more difficult translations. However, still missing from this analysis is a mechanism to consider the fact gaps, confounding facts, and attribute density in respect to both the source and target representations. Additionally missing is some unifying mechanism through which to simultaneously consider all of these dimensions in order to determine which mathematical translation students find most problematic. These missing elements are addressed in following discussions.

\section{Additional Connected Research by the Authors}

Throughout this paper, references are made to a number of studies in which the authors have investigated aspects of interpretive processes students use in respect to mathematical representations and translation processes they implement between mathematical translations. While coherent and complementary in their findings, each study considers a distinct aspect associated with these processes. The following terse descriptions of these studies are expanded upon in proceeding and following discussions as these studies are both connected to research published by others and further placed into the context of the investigation at hand.

Investigating student translation work between numeric, symbolic, and graphical mathematical representations, Adu-Gyamfi et al. (2012), have found three distinct types of common errors that arise and quantify the respective frequency of these error types. Altogether, these three error types encapsulate all possible errors that can occur during the translation process. Expanding upon this study, Adu-Gyamfi et al. (2011b) analyze student activity in the linguistic-to-algebra translation process, define error types made, and recognize the frequencies of such. Comparing and contrasting various explanations for linguistic-to-algebra translation errors, they develop a conceptual framework (TranslationVerification Model) which considers three interrelated constructs defining student actions and understanding and provide a framework through which errors can be characterized and their respective frequencies quantified. The cumulative findings of these two studies regarding translation error types, the frequency of each error type, and the total number of errors 
between each pair of respective representations, provide some insight into the relative difficulty of each translation activity.

Examining student actions, interpretations, and language in respect to questions raised regarding table, graph and symbolic representations in the context of functions, Adu-Gyamfi et al. (2011a) have found that although students may be able to mechanically perform some translations between representations, they may do so without fully understanding salient ideas encoded within each representation. Thus, fully understanding mathematical representations and translating between such are far more complex than can be defined by simple procedural algorithms. Furthermore, Bossé et al. (2011b) found that teacher beliefs, expectations, and instructional practices all work together to provide students experiences leading to differing levels of success in respect to mathematical translations, explaining in part why some translations seem to be more difficult than others.

In two complementary investigations regarding student ability, understanding, and activities in respect to connecting mathematical concepts and performing translations between mathematical representations, the same team of authors has found significant variances between students of differing ability levels. In Adu-Gyamfi, Bossé, and Cheetham (2011c), a framework is developed and applied which allows a deeper analysis of student understanding and actions in respect to the conceptual connection they make across mathematical representations. This framework considers various types of mathematical concepts found within and among representations (micro-concepts, confounding microconcepts, macro-concepts, sub-constructs, infra-constructs, and super-constructs), allows analysis of student understanding of concepts within and between representations, and provides a mapping of how students of different ability levels make different connections among mathematical representations. Bossé et al. (2011a) investigate the translation process itself and map different translation processes among students of different ability levels. Altogether, these studies again provide evidence that, even factoring for differing student ability, some translations between representations are more difficult than other translations.

These research projects assist to construct a more expansive picture of students' understanding and processes in the translating of mathematical representations. These studies, synthesized with numerous studies by other researchers, serve to provide a rubric through which argument can be made regarding the relative level of difficulty can be associated with each translation. This is developed in the following discussions.

\section{Unifying Dimensions and Levels of Difficulty in Mathematical Translations}

With an initial order of difficulty among mathematical translations previously established via student activity (mathematical errors, instructional experiences, interpretive and translation activities, and the use of transitional representations), a simultaneous consideration of fact gaps, confounding facts, and attribute density creates a more complete gestalt explicating the respective level of difficulty of translations. This gestalt simultaneously unifies the extant literature previously discussed and transcends such by generating a more interconnected and complete model through which to both investigate individual translations and solidify an ordering of translations in respect to their respective level of difficulty. 
The symbolic form of the novel interconnected model, depicted in Figure 4, denotes a notably dense coding structure. In the symbolic coding, dimensions of fact gaps, confounding facts, attribute density, translation action, and proximity of interpretive activities are represented.

\begin{tabular}{|ccc|}
\hline $\begin{array}{c}\text { Fact Gaps } \\
\text { Confounding Facts } \\
\begin{array}{c}\text { Source Representation } \\
\text { Attribute Density }\end{array}\end{array} \underset{\text { Translation Action }}{\text { Proximity }}$ & $\begin{array}{c}\text { Fact Gaps } \\
\text { Confounding Facts } \\
\text { Target Representation } \\
\text { Attribute Density }\end{array}$ \\
\hline
\end{tabular}

Figure 4. Multidimensional coding for mathematical translations.

The coding structure in Figure 4 takes into account dimensions that may alter in respect to a representation being either the source or target of the translation and in respect to which translation is being performed. For instance: (1) when the table is a source representation, both its fact gaps and its confounding facts are high. However, as a target, its fact gaps and confounding facts are low. (2) Considering a graph as a source representation, the graph's confounding facts may differ depending on the chosen target representation. (3) As will be further discussed, the characteristics of a table can change as it is used as a transitional representation and changes orientation from target to source.

Figure 5, represented as a table, utilizes the initial findings of level of translation difficulty based on student activity, further analyzes these translations based on additional dimensions, captures the nature of translations utilizing transitional representations, and reorganizes the translations in respect to difficulty. Notably, in this figure, the symbol denotes that in order to perform the translation on the left hand side of $\gg$, students generally perform the translation using the transitional representation as depicted on the right hand side.

The organization and order of the translations in the table (Figure 5) is based upon the recognized difficulty level of each translation, as determined by a multitude of previous studies, and the coding structure in the figure assists in answering why some translations are more difficult than are others. First, as recognized by others (e.g., Duval, 2006; Kaput, 1987; Leinhardt et al., 1990), translation actions which are considered more local are seemingly less difficult than are translation actions which are more global. However, this dimension alone seems only adequate to differentiate easier from more difficult translations and is incapable of further ordering of the difficulty level of each translation.

Second, translations with source representations possessing both fewer fact gaps and fewer confounding facts (table $\rightarrow$ graph, graph $\rightarrow$ table, symbolic $\rightarrow$ table, and verbal $\rightarrow$ table), irrespective of the attribute density, tend to be easier than others. It very well may be that the easiest translations are more an issue of the nature of the source than the target representation. This may be because representations with few fact gaps and few confounding facts are easier to interpret, thus avoiding the error type most commonly recognized early in the translation process, interpretation errors (Adu-Gyamfi et al., 2012).

Third, translations with target representations with a larger number of both fact gaps and confounding facts and low attribute density (graph $\rightarrow$ verbal, symbolic $\rightarrow$ verbal, and table $\rightarrow$ verbal) tend to be far more difficult than all others. (Notably, these equate to 
translations with a verbal representation as their target.) With target representations possessing these characteristics, the translator may tend to feel as if the target is ill defined and amorphous, not know how to get to such an elusive end, and not know when his verbal representation is completed and correct (Bossé et al. (2011a). Therefore, it may also be that the most difficult translations are more an issue of the nature of the target than the source representation.

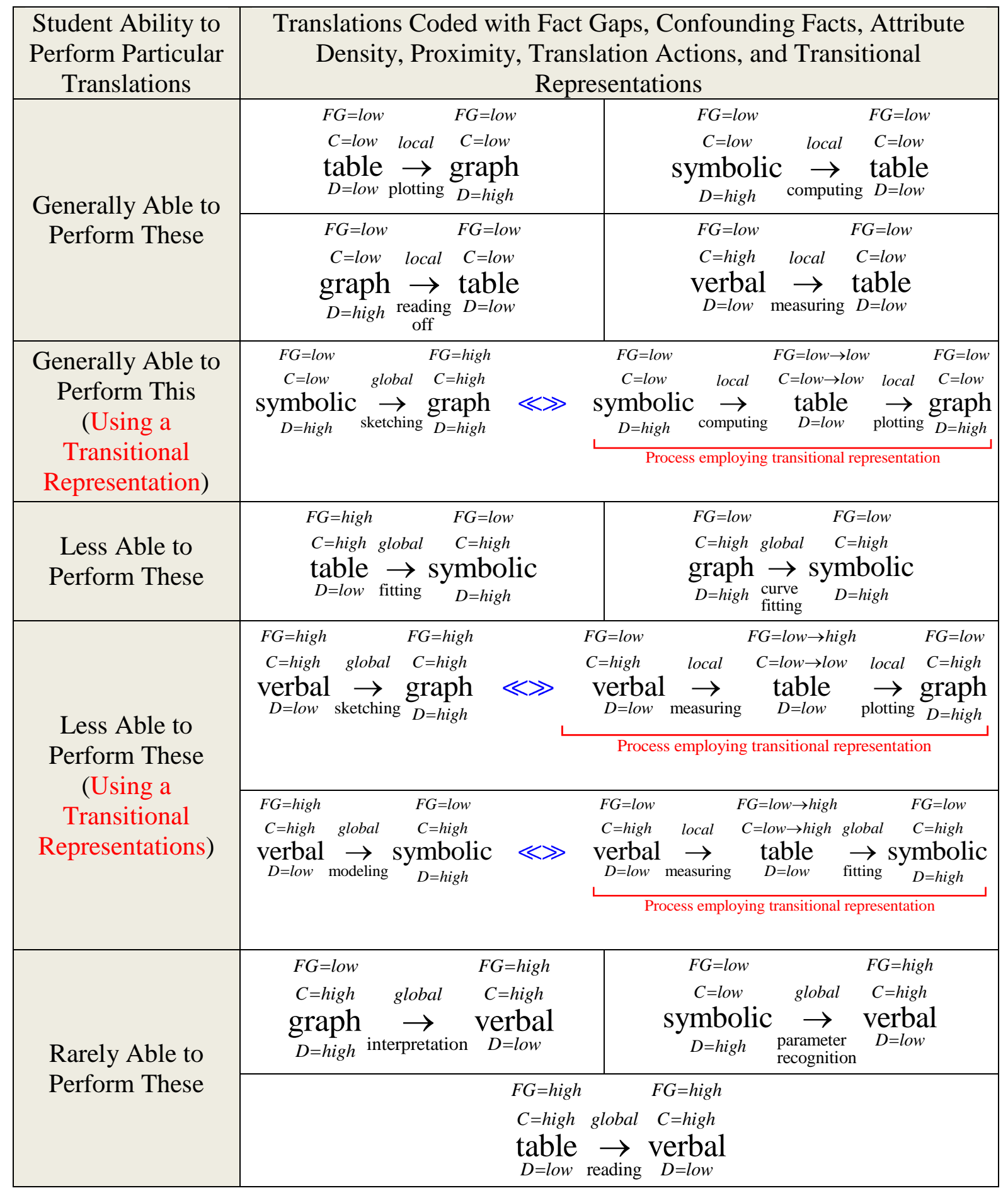

Figure 5. Multidimensional aspects of translations. 
Interestingly, of the twelve possible translations, the five which are recognized as most difficult, all involve the verbal representation as either a source or a target representation (compare with Clement, 1982; Koedinger \& Nathan, 2004). It is hypothesized, herein, that this is because the verbal representation, beyond all others, is more prone to confounding facts. Recognition that the verbal representation possesses characteristic unique among all the representations is noted by many (e.g., Duval, 2006; Kaput, Sims-Knight, \& Clement, 1985; Nathan, Kintsch, \& Young, 1992).

Fourth, this still leaves five translations (symbolic $\rightarrow$ graph, table $\rightarrow$ symbolic, graph $\rightarrow$ symbolic, verbal $\rightarrow$ graph, and verbal $\rightarrow$ symbolic) which lie between the four easiest and the three most difficult translations. Of these five translations, three most often employ transitional representations in the translation process (symbolic $\rightarrow$ graph, verbal $\rightarrow$ graph, and verbal $\rightarrow$ symbolic) and the two most difficult translations (verbal $\rightarrow$ graph and verbal $\rightarrow$ symbolic) have verbal representations as source representations. Notably, the twostep translations utilizing transitional representations break one difficult translation into two simpler translations.

When these two-step translations are dissected into two separate translations, based on the nature and characteristics of the source and target representations and the nature of the translation action, each of these separate translations should not be difficult. Therefore, it may be the simple fact that these are two-step translations, which make them more difficult. It may be because these two-step translations require two separate, yet interconnected translations, and double the work, which makes them more prone to implementation errors (Adu-Gyamfi et al., 2012). However, there may be an additional dimension involved in twostep translations, which further explain why they possess greater complexity.

When transitional representations are employed in a translation, the transitional representation first acts as a target and then as a source in the respective two-step translation process. This changing in the role of the representation carries with it a similar change in the representation's characteristics of fact gaps and confounding facts. For instance, for

\begin{tabular}{|c|c|c|c|c|c|c|c|c|}
\hline$F G=h i g h$ & & $F G=l o w$ & & $F G=$ low & & $F G=l o w \rightarrow$ high & & $F G=l o w$ \\
\hline $\begin{array}{c}C=\text { high } \\
\text { verbal } \\
D=\text { low }\end{array}$ & $\underset{\text { modeling }}{\stackrel{\text { global }}{\rightarrow}}$ & $\begin{array}{c}C=h i g h \\
\text { symbolic } \\
D=h i g h\end{array}$ & $\ll \gg$ & $\begin{array}{l}C=\text { high } \\
\text { verbal } \\
D=\text { low }\end{array}$ & $\stackrel{\text { local }}{\rightarrow} \underset{\text { neasuring }}{\longrightarrow}$ & $\begin{array}{c}C=\text { low } \rightarrow \text { high } \\
\text { table } \\
D=\text { low }\end{array}$ & $\begin{array}{l}\text { global } \\
\underset{\text { fitting }}{\longrightarrow}\end{array}$ & $\begin{array}{l}C=\text { high } \\
\text { ymbolic } \\
D=h i g h\end{array}$ \\
\hline
\end{tabular}

the table representation first acts as a target with characteristics

$$
\begin{gathered}
F G=l o w \\
C=l o w \\
\text { table }
\end{gathered}
$$

and becomes a source with characteristics

$$
\begin{gathered}
\begin{array}{c}
F G=\text { high } \\
C=\text { high } \\
\text { table } \\
D=\text { low }
\end{array} .
\end{gathered}
$$


This change in the characteristics of the representation may be cognitively difficult for students to navigate in the translation process. Indeed, without specific instruction investigating this matter, this phenomenon may always remain tacit and unnavigable by most.

Fifth, since verbal representations are significantly different from other representations, prove to be the most difficult to work with, are less frequently encountered in instructional settings, and much research on translations do not consider the verbal representation, it is valuable to consider the findings of this study independent of verbal representations. With this caveat in place, the delineation of simpler to more difficult translations becomes less complex and is denoted in Figure 6.

$\underbrace{\begin{array}{c}\text { table } \rightarrow \text { graph } \\
\text { graph } \rightarrow \text { table }\end{array}}_{\text {Simplest }} \quad \underbrace{\text { symbolic } \rightarrow \text { table } \rightarrow \text { graph }}_{\text {Less Simple }}$\begin{tabular}{c}
$\begin{array}{c}\text { table } \rightarrow \text { symbolic } \\
\text { graph } \rightarrow \text { symbolic }\end{array}$ \\
$\underbrace{}_{\text {More Difficult }}$ \\
\hline
\end{tabular}

Figure 6. Order of translation difficulty without verbal representations.

With this streamlined structure, discovery of a more generalized predictor of a translation's level of difficulty can be more readily made. It immediately becomes evident that translations with both low fact gaps and low confounding facts are significantly easier and those with both high fact gaps and high confounding facts are significantly more difficult.

Unfortunately, in its current form, this study is unable to make more definitive statements regarding the singular effect of attribute density on translation difficulty or the effects of attribute density as it works in tandem with fact gaps and confounding facts. It is anticipated that future research would further answer these questions.

\section{Discussions, Implications, and Conclusions}

Altogether, this synthesis demonstrates that not all translations are alike and that numerous factors interact to make some translations more difficult than others. Translations involving verbal representations are among the most difficult and representations with fewest fact gaps and confounding facts are involved in translations, which are among the easiest. A cofactor affecting which representations are most difficult is the lack of attention some get during daily classroom instruction.

While numerous previous studies shed some light on which translations may be more difficult than others, this synthesis paints a more complete picture regarding why particular translations are more difficult than others. Characteristics such as instructional practices, translation action, proximity, error types, the use of transitional representations, interacting with verbal representations, fact gaps, confounding facts, and representational density all interact to make some translations more problematic.

The instructional implications from these findings are numerous and significant. In order for teachers to better assist students with mathematical translations, teachers must know which are easier and which are more difficult and provide a sufficient number of experiences 
with the latter to help students gain facility with such. Additionally, teachers must use questioning techniques, which lead students to consider not only what information a representation provides but also what information may be missing in respect to a particular translation, and what information in the source may be confusing or confounding.

With the findings of this paper in mind, teachers should be able to better construct instructional situations through which translation problem scenarios can be more revelatory in respect to student mathematical understanding. More than simply recognizing whether or not students are able to perform a particular translation, teachers may better recognize where in the translation process students stumble, what type of errors they commit, and why they are more likely to commit particular errors on certain translations. In a more positive light, rather than simply assessing student errors, teacher understanding of the nature and difficulty level of respective translations can lead to instructional practices that assist students in correctly interpreting mathematical representations and performing translations.

It is hoped that the more interconnected model for considering the nature of translations between mathematical representations presented in this investigation will assist future research in respect to student actions, understanding, and ability in performing these translations. Beyond simply determining whether or not students are able to perform particular translations, it is hoped that findings herein will allow researchers a deeper and more complete look into student cognition and processes and determine more precisely what students are doing incorrectly, where their difficulties originate, why they struggle, and what instructional experiences can be provided to students to better mitigate problematic issues and cause students to more effectively perform these translations.

\section{References}

Adu-Gyamfi, K., Stiff, L., \& Bossé, M. J. (2012, in press). Lost in translation: Examining translation errors associated with mathematics representations. School Science and Mathematics Journal.

Adu-Gyamfi, K., Bossé, M. J., \& Cheetham, M. (2011a, under review). Situated interpretation: Reasoning and processes in mathematical representation.

Adu-Gyamfi, K., Bossé, M. J., \& Cheetham, M. (2011b, under review). Situating student errors: Linguistic-to-algebra translation errors.

Adu-Gyamfi, K., Bossé, M. J., \& Cheetham, M (2011c, under review). Student connection of mathematical representations: Differentiated by ability.

Bell, A., Brekke, G. and Swan, M. (1987). Diagnostic teaching: 4 graphical interpretations. Mathematics Teaching, 119, 56-60.

Bossé, M. J., Adu-Gyamfi, K., \& Cheetham, M. (2011a, under review). Translation processes differentiated by student ability.

Bossé, M. J., Adu-Gyamfi, K., \& Cheetham, M. (2011b). Translations among mathematical representations: Teacher beliefs and practices. International Journal of Mathematics Teaching and Learning. Retrieved from http://www.cmit.plymouth.ac.uk/journal/default. htm. 
Brenner, M., E, Herman, S., Ho, H., \& Zimmermann, J. (1999). Cross national comparison of representational competence. Journal for Research in Mathematics Education, 30(5), 541-557. doi:10.2307/749773

Carlson, M. P. (1998). A cross sectional investigation of the development of the function concept. In E. Dubinsky, A. Schoenfeld, \& J. Kaput (Eds.), Research in Collegiate Mathematics Education (Vol. 7, pp. 115-162). Providence, RI: American Mathematical Society.

Clement, J., Lockhead, J., \& Monk, G. S. (1981). Translation difficulties in learning mathematics. American Mathematical Monthly, 88, 287-290. doi:10.2307/2320560

Clement, J. (1982). Algebra word problem solutions: Though processes underlying a common misconception. Journal for Research in Mathematics Education, 13(1), 16-30. doi: $10.2307 / 748434$

Cunningham, R. F. (2005). Algebra teachers' utilization of problems requiring transfer between algebraic, numeric and graphic representations. School Science and Mathematics, 105(2), 73-82. doi:10.1111/j.1949-8594.2005.tb18039.x

Dreyfus, T., \& Eisenberg, T. (1987). On the deep structure of functions. In J. C. Bergeron, N. Herscovics, C. Kieran (Eds.). Proceedings of the 11th International Conference for the PME (Vol. I, pp. 190-196). Montreal, Canada.

Duval, R. (2006). A cognitive analysis of problems of comprehension in the learning of mathematics. Educational Studies in Mathematics, 61, 103-131. doi:10.1007/s10649-006$\underline{0400-\mathrm{Z}}$

Dunham, P., H., \& Osborne, A. (1991). Learning how to see: Students' graphing difficulties. Focus on Learning Problems in Mathematics, 13, 35-49.

Gagatsis, A., \& Shiakalli, M. (2004). Ability to translate from one representation of the concept of function to another and mathematical problem solving. Educational Psychology, 24(5), 645-657. doi:10.1080/0144341042000262953

Galbraith, P., \& Haines, C. (2000). Conceptual mis(understandings) of beginning undergraduates. International Journal of Mathematical Education in Science and Technology, 31(5), 651-678. doi:10.1080/002073900434350

Janvier, C. (1987). Translation process in mathematics education. In C. Janvier (Ed.), Problems of representation in mathematics learning and problem solving (pp. 27-31). Hillsdale, NJ: Lawrence Erlbaum Associates.

Kaput, J. J. (1987). Toward a theory of symbol use in mathematics. In C. Janvier (Ed.), Problems of representation in mathematics learning and problem solving (pp. 159-195). Hillsdale, NJ: Erlbaum.

Kaput, J. J. (1989). Linking representations in the symbol systems of algebra. In S. Wagner, \& C. Kieran (Eds.), Research issues in the learning and teaching of algebra (pp. 167194). Hillsdale, NJ: LEA.

Kaput, J., \& Sims-Knight, J. E. (1983). Errors in translations to algebraic equations: Roots and implications. Focus on Learning Problems in Mathematics, 5(3), 63-78.

Kaput, J. J., Sims-Knight, E. J., \& Clement, J. (1985). Behavioral objections: A response to Wollman. Journal for Research in Mathematics Education, 16(1), 56-63. $\underline{\text { doi: } 10.2307 / 748974}$ 
Kerslake, D. (1981), Graphs. In K. Hart (Ed.), Children's understanding of mathematics: 1116 (pp. 120-136). Oxford: John Murray.

Kieran, C. (1993). Functions, graphing, and technology: Integrating research on learning and instruction. In T. Romberg, E. Fennema \& T. Carpenter (Eds.), Integrating research on the graphical representation of functions (pp. 189-237). Hillsdale, NJ: Lawrence Erlbaum Associates.

Knuth, E. J. (2000). Student understanding of the Cartesian connection: An exploratory study. Journal for Research in Mathematics Education, 31(4), 500-508. doi: $10.2307 / 749655$

Koedinger, K. R., \& Nathan, M. J. (2004.). The real story behind story problems: Effects of representations on quantitative reasoning. The Journal of the Learning Sciences, 13(2), 129-164. doi:10.1207/s15327809j1s1302_1

Leinhardt, G., Zaslavsky, O., \& Stein, K. M. (1990). Functions, graphs and graphing: Tasks, learning and teaching. Review of Educational Research, 60(1), 1-64. doi:10.3102/00346543060001001

Lesh, R., Landau, M., \& Hamilton, E. (1983). Conceptual models in applied mathematical problem solving research. In R. Lesh \& M. Landau (Eds.). Acquisition of mathematical concepts and processes (pp. 263-343). New York: Academic Press.

Macgregor, M., \& Stacey, K. (1993). Cognitive models underlying students' formulation of simple linear equations. Journal for Research in Mathematics Education, 24(3), 217-232. doi: $10.2307 / 749345$

Mevarech, Z. \& Kramarsky, B. (1997). From verbal descriptions to graphic representations: stability and change in students alternative conceptions. Educational Studies in Mathematics, 32, 229-263. doi:10.1023/A:1002965907987

Monk, S. (1992). Students' understanding of a function given by a physical model. In G. Harel, \& E. Dubinsky (Eds.), The concept of function: Aspects of epistemology and pedagogy (175-193). Washington, DC: Mathematical Association of America.

Nathan, M. J., Kintsch, W., \& Young, E. (1992). A theory of algebra-word problem comprehension and its implications for the design of learning environments. Cognition and Instruction, 9, 320-389. doi:10.1207/s1532690xci0904_2

Porzio, D. T. (1999). Effects of differing emphasis on the use of multiple representations and technology on students' understanding of calculus concepts. Focus on Learning Problems in Mathematics, 21(3), 1-29.

Preece, J. (1983). Graphs are not straightforward. In T. R. G. Green, \& S. J. Payne (Eds.), The psychology of computer use: A European perspective, (pp. 41-50). Academic Press, London.

Pyke, C. (2003). The use of symbols, words, and diagrams as indicators of mathematical cognition: A causal model. Journal for Research in Mathematics Education, 34, 406-432. doi: $10.2307 / 30034794$

Resnick, L., \& Omanson, S. (1987). Learning to understand arithmetic. In R Glaser (Eds.), Advances in instructional psychology volume 3. (pp. 41-95). Hillsdale, NJ: LEA.

Roth, W. M., \& Bowen, G. M. (2001). Professionals read graphs: A semiotic analysis. Journal for Research in Mathematics Education, 32, 159-194. doi:10.2307/749672 
Schoenfeld, A. H., Smith, J., \& Arcavi, A. (1993). Learning: The microgenetic analysis of one student's evolving understanding of a complex subject matter domain. In R. Glaser (Eds.), Advances in instructional psychology (Vol. 4, pp. 55-175). Hillsdale, NJ:Erlbaum.

Superfine, A. C., Canty, R. S., \& Marshall, A. M. (2009). Translation between external representation systems in mathematics: All-or-none or skill conglomerate? The Journal of Mathematical Behavior, 28(4), 217-236. doi:10.1016/j.jmathb.2009.10.002

Wainer, H. (1992). Understanding graphs and tables. Educational Researcher, 21, 14-23. doi:10.3102/0013189X021001014

Webb, N. M., Gold, K., Qi, S., \& Educational Resources Information Center (U.S.) (1990). Translation between symbolic representations [microform] : relationship between instruction and performance. UCLA Center for Research on Evaluation, Standards, and Student Testing; U.S. Dept. of Education, Office of Educational Research and Improvement, Educational Resources Information Center, Los Angeles, CA.

Wollman, W. (1983). Determining the sources of error in a translation from sentence to equation. Journal for Research in Mathematics Education, 14(3), 169-181. doi: $10.2307 / 748380$

Yerushalmy, M. (1991). Student perceptions of aspects of algebraic function using multiple representation software. Journal of Computer Assisted Learning, 7, 42-57. doi:10.1111/j.1365-2729.1991.tb00223.x

\section{Authors}

Michael J. Bossé, Associate Professor, Department of Mathematics, Science, and Instructional Technology Education, College of Education, East Carolina University, Greenville, NC, 27858, USA; bossem@ecu.edu; +1 (252) 328-9367.

Kwaku Adu-Gyamfi, Assistant Professor, Department of Mathematics, Science, and Instructional Technology Education, College of Education, East Carolina University, Greenville, NC, 27858, USA; adugwamfik@ecu.edu; +1 (252) 328-9369.

Meredith R. Cheetham, M.Sc. Student, Department of Mathematics, Science, and Instructional Technology Education, College of Education, East Carolina University, Greenville, NC, 27858, USA; cheethamm09@students.ecu.edu. 